\title{
Searches for the new magnetic intermediate-mass stars on various stages of MS evolution
}

\author{
Evgeny A. Semenko \\ Special Astrophysical Observatory of the Russian Academy of Sciences, \\ Nizhny Arkhyz, Russia \\ email: sea@sao.ru
}

\begin{abstract}
A limited list of new results of the searches for the new magnetic stars among late $\mathrm{B}$ and early A stars is in this work. Continual observations with spectroscopic devices of the $6 \mathrm{~m}$ Russian telescope BTA led to successful detection of about 10 new magnetic stars that occupy different parts of evolutional tracks for the stars of 2-3 solar masses. Measurements of the longitudinal magnetic field show weak and medium strength magnetic field in all program stars.
\end{abstract}

Keywords. star, magnetic field, spectropolarimetry

\section{Introduction}

The study of stellar magnetism goes on during more than 60 last years, and now more than 450 magnetic Ap/Bp stars are known. Only few dozens of them ever became the objects of detailed study. Better understanding of an evolution of stellar magnetic fields require the extension of the list of known $\mathrm{mCP}$.

The current work is pointed to detection of new magnetic stars among poorly studied B8-A3 stars. By their physical properties these stars are considered as an intermediate class of magnetic stars that occur on different stages of MS evolution.

Below the results of longitudinal field measurements are presented. Most of stars were discovered as magnetic for the first time within the current work during the last 3 years.

\section{Observations. Data reduction}

Spectral material for our work was collected with the Main Stellar Spectrograph (MSS) installed in the Nasmyth-2 focus of the Russian $6 \mathrm{~m}$ telescope BTA. This spectrograph equipped with the differential circular polarization analyzer and allows to observe the stars up to 11-12 magnitude in spectropolarimetric mode. Long-slit spectra have a mean resolution of $0.12 \AA \mathrm{pix}^{-1}$.

The raw data were reduced with a set of programs mentioned by Kudryavtsev et al. (2006). The same set is capable to make the measurements of longitudinal magnetic field by fitting a gaussian function to spectral lines profile. Additionally, using a custom implementation of method, described by Bagnulo et al. (2002) we control the positional magnetic measurements. In order to check the right polarity of magnetic field and take probable instrumental polarization into account we observed every night one or two standard stars with well-measured magnetic field along with cool zero-field star. 


\section{The results}

\subsection{HD 50341}

This star was selected for observation due to its photometric IR excess typical for the young stars. Despite of the low accuracy of measurements, caused by the fast rotation, six individual measurements allow us to make the conservative conclusion about magnetic nature of HD 50341. Further study of this star will consider also the detailed analysis of hi-res spectra.

\section{2. $H D 63347$}

HD 63347 was selected for observation due to its IR excess. Additional indication of its young age was found in Tetzlaff et al. (2011). On the figure our measurements of $B_{z}$ phased with period 1.74984 days (Koen, Eyer 2002). Detailed study of echelle spectra, obtained with BTA, confirm $\mathrm{SrCrEu}$ abundance anomalies that together with young age make this star unique.

\subsection{HD 96003}

Bright star $\left(V=6{ }^{m} 06\right)$ was characterised as SrCr by Renson \& Manfroid (2009). Seven measurements of polarized, rich on the lines, spectra demonstrate approximately constant (about $-150-180 \mathrm{G}$ ) value of $B_{z}$. HD 96003 is a binary system with angular separation of about $2^{\prime \prime}$ between components. An individual measurement of each component should be attractive and may have a high importance for the understanding of stellar magnetic field evolution.

\subsection{HD 201174}

In the current list HD 201174 is the only one early known magnetic star. The first measurements of its longitudinal field were made in SAO RAS by D. Kudryavtsev. Strong cross-over effect and sharp spectral lines allow us to make a detailed study of physical properties of the star. Location of HD 201174 on the HR diagram is typical for young stars. Rotational period is still unknown but 16 individual measurements of $B_{z}$ imply relatively short value of $P_{\text {rot }}$.

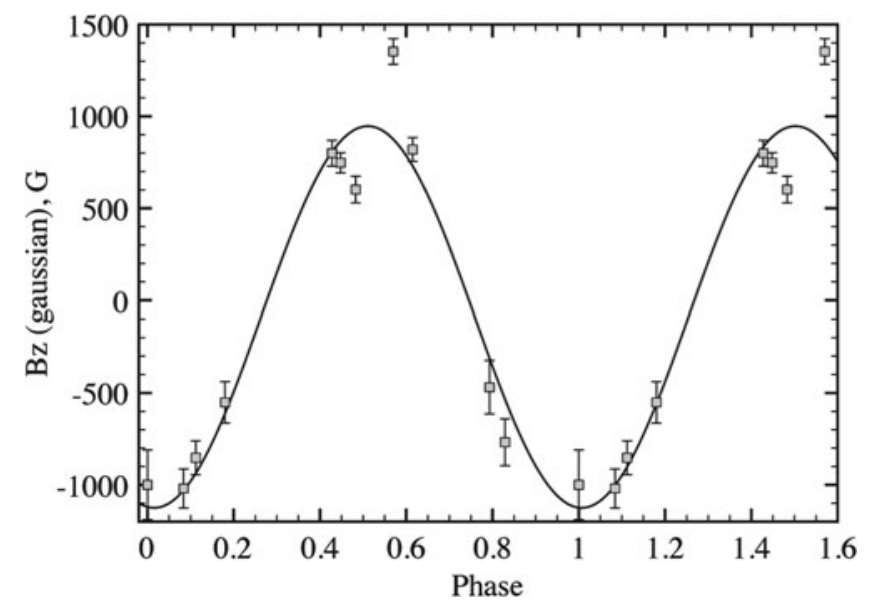

Figure 1. Periodic variability of longitudinal magnetic field of HD 63347. 


\section{Acknowledgments}

The author is grateful to the staff of the Laboratory for stellar magnetism study of the Special Astrophysical Observatory for assistance in collecting observational data and any useful discussions. This work is supported partly by the Russian Fund for Basic Researches (grants RFBR No 12-02-00009-a and 12-02-31246_mol-a) and by the programs of the Ministry of Education and Science of the Russian Federation.

\section{References}

http://www.sao.ru/hq/lizm/mss/en/

Kudryavtsev, D. O., Romanyuk, I. I., Elkin, V. G., \& Paunzen, E. 2006, MNRAS, 372, 1804

Bagnulo, S., Szeifert, T., Wade, G. A., Landstreet, J. D., \& Mathys, G. 2002, A\&A, 389, 191

Renson, P. \& Manfroid, J. 2009, A\&A, 498, 961

Tetzlaff, N., Neuhäuser, R., \& Hohle, M. M. 2011, MNRAS, 410, 190

Koen, C. \& Eyer, L 2002, MNRAS, 331, 45 